\title{
511.
}

\section{ADDITION TO THE MEMOIR ON GEODESIC LINES, IN PARTICULAR THOSE OF A QUADRIC SURFACE (509). $\left({ }^{1}\right.$ )}

[From the Proceedings of the London Mathematical Society, vol. IV. (1871-1873), pp. 368-380. Read June 12, 1873.]

38. IN the Memoir above referred to, speaking of the geodesic lines on the skew hyperboloid, I say (No. 35), "The geodesic of initial direction $M 1$ touches at $M$ the oval curve of curvature $M 1$, and lies wholly above this curve; it makes an infinity of convolutions round the upper part of the hyperboloid, cutting all the oval curves of curvature for which $p$ has a positive value greater than $p_{1}$ (if $p_{1}$ is the value of $p$ corresponding to the oval curve through $M$ ), and ascending to infinity." The statement as to the infinity of convolutions is incorrect; I was led to it by the assumption that the geodesic could not touch any hyperbolic curve of curvature. The fact is, that it touches at infinity (has for asymptotes) in general two hyperbolic curves of curvature; viz. the geodesic descending from infinity in the direction of a hyperbolic curve of curvature, so as to touch the oval curve through $M$, again ascends to infinity in the direction of a hyperbolic curve of curvature (the same as the first-mentioned one, or a different curve), making in its whole course say $k$ convolutions, where $m$ is a positive finite number; if $k<1$, there is no complete convolution, and when $k=1$ or any integer number, then the two hyperbolic curves are one and the same curve; $k$ is infinite only in the special case afterwards referred to in the same No. 35, where the oval curve of curvature is the ellipse which is a principal section of the hyperboloid, and does not even attain to the value 1 except for an oval curve exceedingly close to this ellipse. The error was on consideration obvious enough, though I was in fact led to perceive it by the numerical calculations about to be referred to, which gave me geodesics not making a complete convolution.

1 The articles are numbered consecutively with those of the Memoir, (509). 
39. I have effected, for a particular skew hyperboloid and oval curve of curvature thereof, the numerical calculations for laying down the geodesic lines which touch this curve of curvature. Taking in general the equation of the hyperboloid to be

$$
\frac{x^{2}}{a}+\frac{y^{2}}{b}+\frac{z^{2}}{c}=1,
$$

and the $\theta$-curve of curvature to be the intersection by the confocal surface

$$
\frac{x^{2}}{a-\theta}+\frac{y^{2}}{b-\theta}+\frac{z^{2}}{c-\theta}=1,
$$

then the selected values for the hyperboloid, and oval curve of curvature touched by the geodesics, are

$$
\begin{array}{lr}
a= & 900 \\
b= & 400 \\
c=-c^{\prime}=-1600 \\
\theta=-\theta^{\prime}=-1650
\end{array}
$$

so that $a, b, c^{\prime}, \theta^{\prime}$ are the positive values $900,400,1600$, and 1650 respectively. I recall that $p=c^{\prime}$ to $p=\infty$ gives the oval curves of curvature, viz. $p=c^{\prime}$, the elliptic principal section; $p=\theta^{\prime}$, the given oval curve: and that we are in the sequel concerned only with the oval curves above this, for which $p$ extends from $\theta^{\prime}$ to $\infty$. Moreover, $q=-b$ to $-a$ gives the hyperbolic curves of curvature, viz. $q=-b$ the $x z$-principal section; and $q=-a$ the $y z$-principal section of the hyperboloid. We have, in fact, to deal with the integrals

and

$$
\Pi(p)=\int_{\theta^{\prime}} d p \sqrt{\frac{p}{(p+a)(p+b)\left(p-c^{\prime}\right)\left(p-\theta^{\prime}\right)}},
$$

$$
\Psi(q)=\int_{-b} d q \sqrt{\frac{q}{(q+a)(q+b)\left(q-c^{\prime}\right)\left(q-\theta^{\prime}\right)}} ;
$$

or if $p=\theta^{\prime}+u, u$ extending from 0 towards $\infty$, then

$$
\Pi(p)=\int_{0}^{u} d u \sqrt{\frac{u+\theta^{\prime}}{\left(u+a+\theta^{\prime}\right)\left(u+b+\theta^{\prime}\right)\left(u+\theta^{\prime}-c^{\prime}\right) u}},
$$

and so if $q=-b-v, v$ extending from 0 towards $(a-b)$, which is its limit,

$$
\Psi(q)=\int_{0}^{v} d v \sqrt{\frac{v+b}{(a-b-v) v\left(v+b+c^{\prime}\right)\left(v+b+\theta^{\prime}\right)}} ;
$$

the relation for any particular geodesic of the series being

$$
\pm \Pi(p) \pm \Psi(q)=C \text {. }
$$

40. To avoid discontinuity as to sign, it is convenient to take the integral $\Psi(q)$ in a particular manner. The hyperboloid is by the $x z$ - and $y z$-principal planes divided into four quadrants; or since we attend only to the upper half of the hyperboloid, say this upper half is thus divided into four quadrants, $x$ to $y, y$ to $x^{\prime}, x^{\prime}$ to $y^{\prime}$, and $y^{\prime}$ to $x$; or call them the first, second, third, and fourth quadrants. But we may consider the quadrants as forming an infinite succession, first, second, third, fourth, fifth, 
and so on; or we may take them in the reverse order, $-1,-2,-3$, \&c. For a hyperbolic curve $q=-b-v$ in the first quadrant the integral is to be taken $v=0$ to $v=v$; for a curve in the second quadrant $v=0$ to $a-b$, and thence positively $a-b$ to $v$; for a curve in the third quadrant $v=0$ to $a-b$, thence $a-b$ to 0 , and thence 0 to $v$; and so on: and so for a point in the quadrant -1 , the integral is from 0 to $v$, taken negatively, \&c.; that is, as the hyperbolic curve travels from the $x z$-position in the positive direction, the integral $\Psi(q)$ continually increases from zero; and if the curve travels from the $x z$-position in the negative direction, then the integral $\Psi(q)$ continually decreases from zero; that is, it increases negatively. It is to be remarked that the integral $v=0$ to $a-b$ is finite, say it is $=K^{\prime}$; and of course it is only thus far that the integral requires to be calculated, the subsequent values differing from the preceding ones only by multiples of this complete integral.

The integral $\Pi(p)$ requires no explanation; it is taken from $u=0$, giving a certain oval curve, up to any positive value of $u$, giving the oval curves above this one; and, in particular, taking the integral to $u=\infty$ (or, what is the same thing, to $p=\infty$ ) its value is finite, $=K$, suppose.

41. Consider the geodesic which touches the given oval curve at a point for which $\Psi(q)$ has a given value $Q$; at this point $p=\theta^{\prime}$, or $\Pi(p)=0$; so that, taking for the equation of the geodesic $\Psi(q) \pm \mathrm{II}(p)=C$, we have $Q=C$, and consequently

$$
\Psi(q)=Q \mp \Pi(p) \text {. }
$$

Taking the positive sign, then as $p$ increases from $\theta^{\prime}, \Psi(q)$ increases, or the describing point of the geodesic moves upwards from the point of contact in the direction of positive rotation; and taking the negative sign, then $\Psi(q)$ decreases, or the describing point of the geodesic moves upwards from the point of contact in the direction of negative rotation; and, in particular, $p$ becoming infinite, then the firstmentioned branch touches at infinity the hyperbolic curve, for which $q$ is such that $\Psi(q)=Q+K$, and the second branch that for which $q$ is such that $\Psi(q)=Q-K$.

42. The graphical process is as follows: we describe, on the hyperboloid, a series of hyperbolic curves of curvature, numbering them according to the values of $\Psi(q)$; viz. considering the hyperbolic branches which form the $x z, y z, x^{\prime} z$ and $y^{\prime} z$ sections respectively, these are $0, K^{\prime}, 2 K^{\prime}, 3 K^{\prime}$, and on going round a second time they would be $4 K^{\prime}, 5 K^{\prime}, 6 K^{\prime}, 7 K^{\prime}$, and so on respectively. We similarly describe, say on the upper half of the hyperboloid, the oval curves of curvature, numbering them according to the values of $\Pi(p)$, viz. beginning with that for which $p=\theta^{\prime}$, which is 0 , we go successively up to the oval curve at infinity, which is $K$.

In the example, and drawing belonging thereto $\left({ }^{1}\right)$, where, for convenience, the values of the integrals have been multiplied by 100,000 , we have, as will appear, $K=12490$, $K^{\prime}=34726$ : the two sets of curves are drawn at intervals of 2000 ; viz. we have the hyperbolic curves $0,2000,4000, \ldots 34000,34726$; and the oval curves $0,2000,4000$, $6000,8000,10000$; the distance of the successive oval curves increases very rapidly, since the curve at infinity would be $K,=12490$, and the curve $K=10000$ is the last which comes into the limits of the figure.

1 This drawing was exhibited at the meeting of the Society. 
43. The two sets of curves of curvature being thus drawn at equal intervals of $\Pi(p)$ and $\Psi(q)$ respectively, dividing the hyperboloid into quadrilateral spaces (which of course should theoretically be indefinitely small), the diagonals of these quadrilateral spaces are the elements of the geodesic lines; and by a series of such elements we have a particular geodesic line. The general character comes out in the drawing very distinctly; viz. the geodesic is a hyperbola-like curve descending from infinity to touch the oval curve, and again ascending to infinity; by reason of the small value of $K$ in comparison of $K^{\prime}$, there is nothing like a complete convolution, but the whole curve is included within a quadrant of the hyperboloid.

44. I remark that the calculations were performed roughly. I made no attempt to estimate or allow for errors arising from the intervals being too great; and there are very probably accidental errors of calculation. But starting with the value 10411 of $\Pi(p)$ for $p=10000$, I found, with some care, superior and inferior limits of the remainder of the integral, $p=10000$ to $p=\infty$; and the process is, I think, an interesting one. Consider in general the integral

$$
\begin{aligned}
I & =\int_{\theta^{\prime}}^{\infty} d p \sqrt{\frac{p}{(p+a)(p+b)\left(p-c^{\prime}\right)\left(p-\theta^{\prime}\right)}} \\
& =I_{1}+\int_{p_{1}}^{\infty} d p \sqrt{\frac{p}{(p+a)(p+b)\left(p-c^{\prime}\right)\left(p-\theta^{\prime}\right)}},
\end{aligned}
$$

where $I_{1}$ is the integral calculated up to a somewhat large value $p=p_{1}$.

Writing

$$
\begin{aligned}
& \alpha=\frac{1}{2}(a+b), \\
& \beta=\frac{1}{2}\left(c^{\prime}+\theta^{\prime}\right), \\
& \gamma=\frac{1}{2}(a+b)-m, \\
& \delta=\frac{1}{2}\left(c^{\prime}+\theta^{\prime}\right)+n,
\end{aligned}
$$

where $m$ and $n$ are as yet undetermined, we have

$$
\begin{array}{ll}
(p+a)(p+b)=(p+\alpha)^{2}-\frac{1}{4}(a-b)^{2}, & >(p+\alpha)^{2}, \\
\left(p-c^{\prime}\right)\left(p-\theta^{\prime}\right)=(p-\beta)^{2}-\frac{1}{4}\left(\theta^{\prime}-c^{\prime}\right)^{2}, & >(p-\beta)^{2} ;
\end{array}
$$

and the integral is thus

$$
>I_{1}+\int_{p_{1}}^{\infty} d p \frac{\sqrt{ } p}{(p+\alpha)(p-\beta)} .
$$

But we may determine $m$ and $n$, so that for $p=$ or $>p_{1}$,

and the integral is then

$$
\begin{aligned}
& (p+a)(p+b)<(p+\gamma)^{2}, \\
& \left(p-c^{\prime}\right)\left(p-\theta^{\prime}\right)<(p-\delta)^{2} ;
\end{aligned}
$$

$$
<I_{1}+\int_{p_{1}}^{\infty} d p \frac{\sqrt{ } p}{(p+\gamma)(p-\delta)} .
$$

The determination thus depends on the last-mentioned integrals, the values of which are at once obtainable by writing therein $\sqrt{ } p=x$; viz. we have

$$
\int d p \frac{\sqrt{ } p}{(p+\alpha)(p-\beta)}=2 \int \frac{x^{2} d x}{\left(x^{2}+\alpha\right)\left(x^{2}-\beta\right)}=C+\frac{2}{\alpha+\beta}\left\{\frac{1}{2} \beta h \cdot l \frac{x-\sqrt{ } \beta}{x+\sqrt{ } \beta}\right\}+\frac{2 \sqrt{ } \alpha}{\alpha+\beta} \tan ^{-1} \frac{x}{\sqrt{ } \alpha} ;
$$


and hence, substituting in the formula, for h.l.x its value $\frac{\log x}{\log e}$, the superior limit is

$$
I_{1}+\left\{\frac{\sqrt{ } \delta}{\gamma+\delta} \frac{1}{\log e} \log \frac{\sqrt{ } p_{1}+\sqrt{ } \delta}{\sqrt{ } p_{1}-\sqrt{ } \delta}+\frac{2 \sqrt{ } \gamma}{\gamma+\delta} \cot ^{-1} \frac{\sqrt{ } p_{1}}{\sqrt{ } \gamma_{1}}\right\},
$$

and the inferior limit is

$$
I_{1}+\left\{\frac{\sqrt{ } \beta}{\alpha+\beta} \frac{1}{\log e} \log \frac{\sqrt{ } p_{1}+\sqrt{ } \beta}{\sqrt{ } p_{1}-\sqrt{ } \beta}+\frac{2 \sqrt{ } \alpha}{\alpha+\beta} \cot ^{-1} \frac{\sqrt{ } p_{1}}{\sqrt{ } \alpha_{1}}\right\} .
$$

45. The numerical values are $p_{1}=10,000 ; a, b, c^{\prime}, \theta^{\prime}=900,400,1600,1650$; and thence determining by trial values of $m$ and $n$,

$$
\begin{array}{ll}
\alpha=650, & \gamma=650-4=646, \\
\beta=1625, & \delta=1625+160=1785,
\end{array}
$$

I obtained for the logarithmic and circular terms of the two limits respectively

$\begin{array}{lcc}\text { Logarithmic } & \text { Superior } & \text { Inferior } \\ \text { Circular } & .015668 & .015144 \\ & .005202 & .005593 \\ & .020870 & .020737\end{array}$

The value of $I_{1}$ was $10411 \div 100,000=\cdot 104110$, and the two limits thus are 124980 and 124850 ; or restoring the factor 100,000 , they are 12498 and 12485 ; the mean of these, say 12490, was taken for the value $\Pi(p), p=\propto$; that is $K=12490$.

46. As regards the calculation of the integrals $I(p)$ and $\Psi(q)$, introducing the numerical values, and multiplying by the before-mentioned factor 100,000 , we have $(q=-400-v)$,

$$
\Psi(q)=100,000 \int_{0}^{v} d v \sqrt{\frac{v+400}{(500-v) v(v+2000)(v+2050)}},
$$

which for any small value of $v$ is

viz. this is

$$
=100,000 \sqrt{\frac{400}{500 \cdot 2000 \cdot 2050}}\left(\int \frac{d v}{\sqrt{ } v},=2 \sqrt{ } v\right) ;
$$

$$
=883 \cdot 45(\log =2 \cdot 9461830) \sqrt{ } v,
$$

which was used for the values $v=1,2, \ldots 10$, that is, to $q=-410$; after which the calculation was continued by quadratures giving to $v$ the values $10,20,30, \ldots$ up to $v=490$, or $q=-890$. For the remainder of the integral, writing $500-v=w$ (that is, $q=-900+w)$, we have

$$
\begin{aligned}
\Psi(q)-\Psi(-890) & =100,000 \int d w \sqrt{\frac{900-w}{w(500-w)(2500-w)(2550-w)}}, \\
& =100,000 \sqrt{\frac{900}{500 \cdot 2500 \cdot 2550}\left\{\int \frac{d w}{\sqrt{ } w},=2(\sqrt{ } 10-\sqrt{ } w)\right\}} \\
& =1062 \cdot 7(\log =3 \cdot 0264261)(\sqrt{ } 10-\sqrt{ } w),
\end{aligned}
$$


which was used for the values $w=9,8,7, \ldots 1,0$, to complete the calculation up to $q=-900$.

47. We have in like manner, $p=1650+u$,

$$
\Pi(p)=100000 \int_{0}^{u} d u \sqrt{\frac{u+1650}{(u+2550)(u+2050)(u+50) u}},
$$

which for small values of $u$ is

viz. this is

$$
=100000 \sqrt{\frac{1650 .}{2550.2050 .50}}\left(\int \frac{d u}{\sqrt{u}},=2 \sqrt{ } u\right) ;
$$

$$
502 \cdot 5(\log =2 \cdot 7011399) \sqrt{ } u \text {, }
$$

used for $u=1,2, \ldots 10$, that is to $p=1660$. The calculation was afterwards continued by quadratures, by giving to $u$ a succession of values, at intervals at first of 10 , and afterwards of $20,50,100,200$, and 500 , up to $p=10,000$, giving for the integral the value 10411; and thence, as appearing above, the value for $p=\infty$ was found to be $=12490$.

48. After the calculation of the values of $\Pi(p)$ and $\Psi(q)$, it was easy by interpolation to revert these tables, so as to obtain a table which, for $\Pi$ or $\Psi$ as argument, gives the values of $p$ and $q$. The arguments are taken at intervals of 500 ; up to 10000 as regards $p$, since the original table was only calculated thus far; and up to 34726 as regards $q$. I had thus calculated the annexed Table III., when it occurred to me that there was a convenience in taking the arguments to be submultiples of the complete integral 34726 ; say we divide this into 90 parts, or, as it were, graduate the quadrant of the hyperboloid by means of hyperbolic curves of curvature adapted for the geodesics in question. Taking every fifth part, or in fact dividing the quadrant into 18 parts, we have the Table IV.

49. It will be remembered that the foregoing results apply only to the geodesics which touch the oval curve of curvature $p=+1650$; for the geodesics touching any other oval curve of curvature, the values of the integrals, and the mutual distances of the curves of curvature used for tracing the geodesics, would be completely altered. But it is possible to derive some general conclusions as to the geodesics that touch a given oval curve of curvature.

Observe that the integral $K^{\prime}(=34726$ in the case considered) measures the quadrant of the hyperboloid; viz. $\Psi(q)=0, \Psi(q)=K^{\prime}$ determine two hyperbolic curves of curvature (principal sections), the mutual distance whereof is a quadrant. Each geodesic touches the given oval curve of curvature, and it touches at infinity the two hyperbolic curves $\Psi(q)=Q \pm K(K=12490$ in the case considered); viz. the distance of these in regard to the circuit of four quadrants, or say the amplitude of the geodesic, is measured by the ratio $\frac{K}{2 K^{\prime}}$.

50. Now it is easy to see that as the oval curve of curvature approaches the principal elliptic section, that is, as $\theta^{\prime}$ approaches $c^{\prime}$ (or writing $\theta^{\prime}=c^{\prime}+m$, as $m$ c. VIII. 
diminishes towards zero), the integral $K^{\prime}$ alters its value only slowly, increasing towards a certain constant limit; but, contrariwise, $K$ increases without limit, its value for any small value of $m \iota$ being of the form $A-B \log m,=\infty$ in the limit; wherefore, as $m$ diminishes, the value of $\frac{K}{2 K^{\prime}}$, the amplitude of the geodesic, continually increases. If this is $=1$, the geodesic touching at infinity a certain hyperbolic curve of curvature, in descending to touch the oval curve, makes round the hyperboloid a half-convolution, and then again ascends through another half-convolution to touch at infinity the same hyperbolic curve of curvature; viz. it makes in all one entire convolution, or say in descending it makes a half-convolution. But if $K \div 2 K^{\prime}=2$, then the curve makes in descending a complete convolution; and so, if $K \div 2 K^{\prime}=2 s$, then the geodesic makes in descending $s$ convolutions; and, as already mentioned, ultimately when $m=0$ the geodesic makes an infinity of convolutions; that is, it never actually reaches the elliptic principal section, but has this line for an asymptote.

51. To sustain the foregoing statements, I write $\theta^{\prime}\left(=c^{\prime}+m\right)=1600+m$, and I consider the integral

$$
K_{m}^{\prime}=100000 \int_{0}^{\infty} d u \sqrt{\frac{u+1600+m}{(u+2500+m)(u+2000+m)(u+m) u}},
$$

say for a moment this is

$$
K_{m}^{\prime}=\int_{0}^{\infty} U_{m} d u
$$

Supposing $m$ to be small, we divide the integral into two parts, say from 0 to $\alpha$ [where $\alpha,=$ for example 50 or 100 , is large in comparison with $m$, but small in comparison with the numbers $\left(c^{\prime}, \&\right.$ c. $), 1600$, \&c.], and from $\alpha$ to $\infty$. In the second part, the expression under the integral sign and the value of the integral varies slowly with $m$, and we may, as an approximation, write $m=0$. We have thus

$$
K_{m}=\int_{0}^{a} U_{m} d u+\int_{a}^{\infty} U_{0} d u,
$$

and the first part hereof is

$$
=100000 \sqrt{(2500 \cdot 2000}) \int_{0}^{a} \frac{d u}{\sqrt{u(u+m)}}
$$

viz. the integral is here

$$
h . l .\left\{u+\frac{1}{2} m+\sqrt{u(u+m)\}}=h . l . \frac{\alpha+\frac{1}{2} m+\sqrt{\alpha(\alpha+m)}}{\frac{1}{2} m}=h . l . \frac{4 \alpha}{m}\right. \text { approximately }
$$

or say this is

The first term is thus

$$
=\frac{1}{\log e} \log \frac{4 \alpha}{m} .
$$

which is

$$
=100000 \sqrt{\frac{1600}{2500.2000}} \frac{1}{\log e} \log \frac{4 \alpha}{m},
$$

$$
=4119 \log \frac{4 \alpha}{m} \text {, }
$$


or we have

$$
K_{m}^{\prime}=4119 \log \frac{4 \alpha}{m}+\int_{a}^{\infty} U_{0} d u .
$$

We ought to have the same value of the integral, whatever, within proper limits, the assumed value of $\alpha$ may be. Taking, for instance, $\alpha=50$ and $\alpha=100$, we ought to have

that is,

$$
\begin{aligned}
K_{m}^{\prime} & =4119 \log \frac{400}{m}+\int_{100}^{\infty} U_{0} d u \\
& =4119 \log \frac{200}{m}+\int_{50}^{\infty} U_{0} d u ;
\end{aligned}
$$

$$
4119 \log 2=\int_{50}^{100} U_{0} d u .
$$

In verification, I calculated the second side by quadratures; viz. for the values $50,60,70,80,90,100$, the values of $U_{0}$ are $35.532,29.570,25.311,22.373,19 \cdot 632$, 17.645 ; whence, adding the half sum of the extreme terms to the sum of the mean terms, and multiplying by 10 , the value of the integral is $=123474$. The value of the left-hand side is $=1239 \cdot 94$, which is a sufficient agreement.

52. Returning to the formula for $K_{m}^{\prime}$, this may be written

$$
K_{m}^{\prime}=\left(4119 \log 400+\int_{100}^{\infty} U_{0} d u\right)-4119 \log m .
$$

I did not calculate the value of the integral in this formula, but determined the term in ( ) in such wise that the formula should be correct for the foregoing value $m=50$; viz. the term thus is

$$
=12490+4119 \log 50=12490+6998,=19488, \text { or say } 19500 ;
$$

we thus have

$$
K_{m}=19500-4119 \log m \text {; }
$$

and we may roughly assume that, for any small value of $m, K_{m}^{\prime}$ has the same value as for $m=50$; viz. we may write

$$
K_{m}^{\prime}=34726 \text {, or say }=35000 .
$$

We thus see how to give to $m$ such a value that the quantity $\frac{K}{2 K^{\prime}}$, which is the number of convolutions of the geodesic, may have any given value; and, in particular, we see how exceedingly small $m$ must be for any moderately large number of convolutions; for instance, $m=\frac{1}{100,000000}$ or $\log m=-8, K=19500+32952$, = say 52500 , or the number is $=\frac{525}{700}$, about five-sevenths of a convolution.

CoRrection. Instead of speaking, as above, of a geodesic as touching at infinity a hyperbolic curve of curvature, the accurate expression is that the geodesic at infinity is parallel to a certain hyperbolic curve of curvature. The geodesic has, in fact, for asymptote the right line on the surface parallel at infinity to such curve of curvature. Added Dec. 1873. 
TABLe I.

\begin{tabular}{|c|c|c|c|c|}
\hline$p$ & $\Pi(p)$ & & $q$ & $\Psi(q)$ \\
\hline 1650 & 000 & & -400 & 000 \\
\hline 1 & 502 & & 1 & 883 \\
\hline 2 & 711 & & 2 & 1249 \\
\hline 3 & 870 & & 3 & 1530 \\
\hline 4 & 1005 & & 4 & 1767 \\
\hline 5 & 1124 & & 5 & 1975 \\
\hline 6 & 1231 & & 6 & 2164 \\
\hline 7 & 1329 & & 7 & 2337 \\
\hline 8 & 1421 & & 8 & 2499 \\
\hline 9 & 1507 & & 9 & 2650 \\
\hline 1660 & 1589 & & 410 & 2794 \\
\hline 70 & 2188 & & 20 & 4016 \\
\hline 80 & 2605 & & 30 & 4952 \\
\hline 90 & 2933 & & 40 & 5752 \\
\hline 1700 & 3205 & & 50 & 6467 \\
\hline 10 & 3438 & & 60 & 7124 \\
\hline 20 & 3642 & & 70 & 7739 \\
\hline 30 & 3824 & & 80 & 8321 \\
\hline 40 & 3989 & & 90 & 8877 \\
\hline 50 & 4130 & 10 & 500 & 9413 \\
\hline 60 & 4287 & & 10 & 9931 \\
\hline 70 & 4414 & & 20 & 10436 \\
\hline 80 & 4532 & & 30 & 10923 \\
\hline 90 & 4642 & & 40 & 11406 \\
\hline 1800 & 4745 & & 50 & 11880 \\
\hline 20 & 4945 & & 60 & 12347 \\
\hline 40 & 5106 & & 70 & 12809 \\
\hline 60 & 5261 & & 80 & 13266 \\
\hline 80 & 5403 & & 90 & 13720 \\
\hline 1900 & 5533 & & 600 & 14171 \\
\hline 20 & 5654 & & 10 & 14619 \\
\hline 40 & 5766 & & 20 & 15067 \\
\hline 60 & 5871 & & 30 & 15513 \\
\hline 80 & 5970 & & 40 & 15960 \\
\hline 2000 & 6063 & & 50 & 16408 \\
\hline 50 & 6275 & & 60 & 16857 \\
\hline 100 & 6462 & & 70 & 17308 \\
\hline 150 & 6629 & & 80 & 17762 \\
\hline 200 & 6779 & & 90 & 18220 \\
\hline 250 & 6916 & & 700 & 18682 \\
\hline 300 & 7041 & & 10 & 19149 \\
\hline 350 & 7156 & & 20 & 19623 \\
\hline 400 & 7262 & & 30 & 20101 \\
\hline 4.50 & 7362 & WWW.ICII.01g. & -40 & 20588 \\
\hline
\end{tabular}

TABLE II. 
TABLE I. (continued).

\begin{tabular}{|r|r|}
\hline \multicolumn{1}{|c|}{$p$} & $\Pi(p)$ \\
\hline 2500 & 7454 \\
600 & 7625 \\
700 & 7777 \\
800 & 7913 \\
900 & 8037 \\
3000 & 8151 \\
200 & 8352 \\
400 & 8526 \\
600 & 8679 \\
800 & 8815 \\
4000 & 8936 \\
500 & 9216 \\
5000 & 9423 \\
500 & 9593 \\
6000 & 9737 \\
500 & 9861 \\
7000 & 9970 \\
500 & 10066 \\
8000 & 10152 \\
500 & 10229 \\
9000 & 10299 \\
500 & 10363 \\
10000 & 10411 \\
$\vdots$ & \\
50 & 12490 \\
& \\
\hline & \\
500 \\
500
\end{tabular}

TABLE II. (continued).

\begin{tabular}{|r|r|}
\hline \multicolumn{1}{|c|}{$\Psi(q)$} \\
-750 & 21086 \\
60 & 21595 \\
70 & 22117 \\
80 & 22653 \\
90 & 23206 \\
800 & 23778 \\
10 & 24374 \\
20 & 24998 \\
30 & 25655 \\
40 & 26355 \\
50 & 27105 \\
60 & 27928 \\
70 & 28861 \\
80 & 29936 \\
890 & 31365 \\
1 & 31538 \\
2 & 31720 \\
3 & 31914 \\
4 & 32123 \\
5 & 32350 \\
6 & 32600 \\
7 & 32886 \\
8 & 33224 \\
9 & 33663 \\
-900 & 34726 \\
& \\
\hline
\end{tabular}

TABLE III.

Diff.
Diff.

$p$

\begin{tabular}{|r|c|c|r|r|}
\hline 0 & $1650 \cdot 0$ & 1 & $-400 \cdot 0$ & $\cdot 3$ \\
500 & $1651 \cdot 0$ & 3 & $400 \cdot 3$ & $1 \cdot 0$ \\
1000 & $1654 \cdot 0$ & 5 & $401 \cdot 3$ & $1 \cdot 6$ \\
1500 & $1659 \cdot 0$ & $7 \cdot 8$ & $402 \cdot 9$ & $2 \cdot 2$ \\
2000 & $1666 \cdot 8$ & $10 \cdot 7$ & $405 \cdot 1$ & $2 \cdot 9$ \\
2500 & $1677 \cdot 5$ & $14 \cdot 9$ & $408 \cdot 0$ & $3 \cdot 7$ \\
3000 & $1692 \cdot 4$ & $20 \cdot 6$ & $411 \cdot 7$ & $4 \cdot 0$ \\
3500 & $1713 \cdot 0$ & $27 \cdot 8$ & $415 \cdot 7$ & $4 \cdot 3$ \\
4000 & $1740 \cdot 8$ & $36 \cdot 6$ & $420 \cdot 0$ & $5 \cdot 2$ \\
4500 & $1777 \cdot 4$ & $49 \cdot 4$ & $425 \cdot 2$ & $5 \cdot 4$ \\
5000 & $1826 \cdot 8$ & $68 \cdot 1$ & $430 \cdot 6$ & $6 \cdot 2$ \\
5500 & $1894 \cdot 9$ & $91 \cdot 5$ & $436 \cdot 8$ & $6 \cdot 6$ \\
6000 & $1986 \cdot 4$ & $124 \cdot 6$ & $-443 \cdot 4$ & $7 \cdot 1$
\end{tabular}


TABLE III. (continued).

\begin{tabular}{|c|c|c|c|c|}
\hline II or $\Psi$ & $p$ & Diff. & $q$ & Diff. \\
\hline 6500 & 2111 & $124 \cdot 6$ & $-450 \cdot 5$ & $7 \cdot 1$ \\
\hline 7000 & 2283 & 172 & $458 \cdot 1$ & $7 \cdot 6$ \\
\hline 7500 & 2527 & 244 & $466 \cdot 1$ & $8 \cdot 0$ \\
\hline 8000 & 2870 & 353 & $474 \cdot 5$ & $8 \cdot 4$ \\
\hline 8500 & 3285 & 415 & $483 \cdot 2$ & $8 \cdot 7$ \\
\hline 9000 & 4114 & 829 & $49 \cdot \mathbf{2} \cdot 3$ & $9 \cdot 1$ \\
\hline 9500 & 5226 & 1112 & $501 \cdot 7$ & $9 \cdot 4$ \\
\hline 10000 & 7156 & 1930 & $511 \cdot 3$ & $9 \cdot 6$ \\
\hline 500 & . & & $521 \cdot 1$ & $9 \cdot 8$ \\
\hline 11000 & . & & $531 \cdot 5$ & $10 \cdot 1$ \\
\hline 500 & . & & $542 \cdot 0$ & 10.5 \\
\hline 12000 & . & & $552 \cdot 5$ & $10 \cdot 8$ \\
\hline 500 & $\infty$ & & $563 \cdot 3$ & $10 \cdot 8$ \\
\hline 13000 & & & $574 \cdot 2$ & $10 \cdot 9$ \\
\hline 500 & & & $585 \cdot 1$ & $10 \cdot 9$ \\
\hline 14000 & & & $596 \cdot 2$ & $11 \cdot 1$ \\
\hline 500 & & & $607 \cdot 3$ & $11 \cdot 1$ \\
\hline 15000 & & & 618.5 & $11 \cdot 2$ \\
\hline 500 & & Fet & $629 \cdot 7$ & $11 \cdot 2$ \\
\hline 16000 & & H & $641 \cdot 0$ & $11 \cdot 3$ \\
\hline 500 & & & $652 \cdot 1$ & $11 \cdot 1$ \\
\hline 17000 & & & $663 \cdot 2$ & $11 \cdot 1$ \\
\hline 500 & & & $674 \cdot 2$ & $11 \cdot 0$ \\
\hline 18000 & & & $685 \cdot 2$ & $11 \cdot 0$ \\
\hline 500 & & & $696 \cdot 1$ & $10 \cdot 9$ \\
\hline 19000 & & & $706 \cdot 8$ & $10 \cdot 7$ \\
\hline 500 & & & $717 \cdot 4$ & $10 \cdot 6$ \\
\hline 20000 & & & $728 \cdot 0$ & $10 \cdot 6$ \\
\hline 500 & & & $738 \cdot 4$ & $10 \cdot 4$ \\
\hline 21000 & & & $748 \cdot 3$ & $9 \cdot 9$ \\
\hline 500 & & & $758 \cdot 1$ & $9 \cdot 8$ \\
\hline 22000 & & & $767 \cdot 7$ & $9 \cdot 6$ \\
\hline 500 & & & $777 \cdot 4$ & $9 \cdot 4$ \\
\hline 23000 & & & $786 \cdot 4$ & $9 \cdot 0$ \\
\hline 500 & & & $795 \cdot 1$ & $8 \cdot 7$ \\
\hline 24000 & & & $803 \cdot 7$ & $8 \cdot 6$ \\
\hline 500 & & & $812 \cdot 0$ & $8 \cdot 3$ \\
\hline 25000 & & & $820 \cdot 0$ & $8 \cdot 0$ \\
\hline 500 & & & $827 \cdot 6$ & $7 \cdot 6$ \\
\hline 26000 & & & $834 \cdot 9$ & $7 \cdot 3$ \\
\hline 500 & & & $-841 \cdot 9$ & $7 \cdot 0$ \\
\hline
\end{tabular}


TABLE III. (continued).

$\Pi$ or $\Psi \quad p \quad$ Diff.

$q$

Diff.

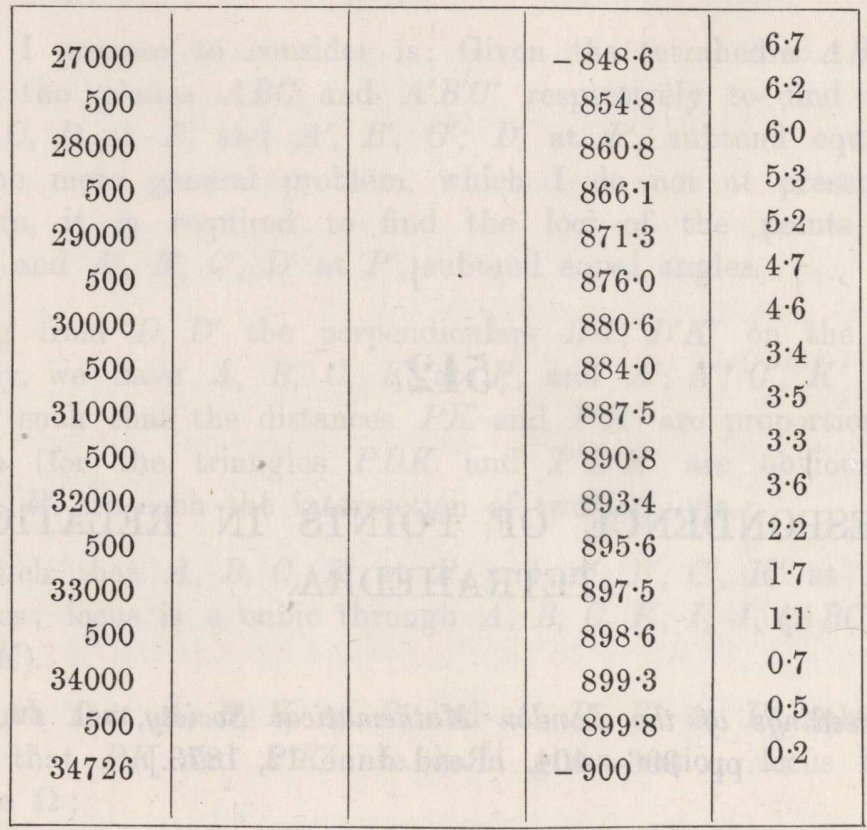

Table IV.

$\begin{array}{lllll}\Pi \text { or } \Psi & p & \text { Diff. } & q & \text { Diff. }\end{array}$

\begin{tabular}{|r|r|r|r|r|r|}
\hline 0 & 0 & 1650 & & & \\
1 & 1929 & $1665 \cdot 7$ & $15 \cdot 7$ & -400 & $4 \cdot 8$ \\
2 & 3858 & $1732 \cdot 1$ & $66 \cdot 4$ & $404 \cdot 8$ & $13 \cdot 9$ \\
3 & 5788 & $1944 \cdot 2$ & $212 \cdot 1$ & $418 \cdot 7$ & $21 \cdot 8$ \\
4 & 7717 & $2657 \cdot 7$ & $713 \cdot 5$ & $440 \cdot 5$ & $29 \cdot 1$ \\
5 & 9646 & $5697 \cdot 7$ & $3040 \cdot 0$ & $469 \cdot 6$ & $34 \cdot 9$ \\
6 & 11575 & & & $504 \cdot 5$ & $39 \cdot 1$ \\
7 & 13504 & & & $543 \cdot 6$ & $41 \cdot 6$ \\
8 & 15434 & & & $585 \cdot 2$ & $42 \cdot 8$ \\
9 & 17363 & & & $628 \cdot 0$ & $43 \cdot 2$ \\
10 & 19292 & & & $713 \cdot 2$ & $41 \cdot 8$ \\
11 & 21221 & & & $752 \cdot 7$ & $39 \cdot 7$ \\
12 & 23150 & & & $789 \cdot 0$ & $36 \cdot 3$ \\
13 & 25079 & & & $821 \cdot 2$ & $32 \cdot 2$ \\
14 & 27008 & & & $848 \cdot 6$ & $27 \cdot 4$ \\
15 & 28938 & & & $870 \cdot 7$ & $22 \cdot 1$ \\
16 & 30868 & & & $886 \cdot 5$ & $15 \cdot 8$ \\
17 & 32797 & & & $896 \cdot 7$ & $10 \cdot 2$ \\
18 & 34726 & & & & \\
& & & & & \\
\hline
\end{tabular}

